\title{
Qubits based on Polariton Rabi Oscillators
}

\author{
S.S. Demirchyan, ${ }^{1}$ I.Yu. Chestnov, ${ }^{1}$ A.P. Alodjants, ${ }^{1,2}$ M.M. Glazov, ${ }^{3,4}$ and A.V. Kavokin ${ }^{4,5}$ \\ ${ }^{1}$ Department of Physics and Applied Mathematics, \\ Vladimir State University named after A.G. and N.G. Stoletovs, Vladimir, 600000, Russia \\ ${ }^{2}$ Russian Quantum Center, Novaya str. 100, 143025, Skolkovo, Moscow, Russia \\ ${ }^{3}$ Ioffe Physical-Technical Institute of the RAS, 26 Polytekhnicheskaya, St.-Petersburg 194021, Russia \\ ${ }^{4}$ Spin Optics Laboratory, St. Petersburg State University, \\ 1 Ul'anovskaya, Peterhof, St. Petersburg 198504, Russia \\ ${ }^{5}$ School of Physics and Astronomy, University of Southampton, SO17 1NJ, Southampton, UK
}

\begin{abstract}
We propose a novel physical mechanism for creation of long lived macroscopic exciton-photon qubits in semiconductor microcavities with embedded quantum wells in the strong coupling regime. The polariton qubit is a superposition of lower branch (LP) and upper branch (UP) exciton-polariton states. We argue that the coherence time of Rabi oscillations can be dramatically enhanced due to their stimulated pumping from a permanent thermal reservoir of polaritons. We discuss applications of such qubits for quantum information processing, cloning and storage purposes.
\end{abstract}

Introduction. - Polaritonics is an interdisciplinary research area at the boundary of optics and solid state physics. It is aimed at the studies of light-matter interaction and dynamics of exciton-polaritons, or shortly polaritons: quasi-particles with bosonic statistics formed as a result of the light-exciton coupling. Nowadays polaritonics represents an indispensable tool for investigation of quantum coherent and nonlinear phenomena occurring at the matter-field interface in various area of condensed matter physics, quantum and atom optics [1-4].

Semiconductor microcavities serve as a solid-state laboratory to study dynamical and quantum effects in open and non-equilibrium systems of bosons. Particularly, one of the main achievements in the field of polaritonics is the creation of and manipulation with condensates characterized by a macroscopic occupation of a single quantum state and extended temporal and spatial coherences. In this sense, polaritonics presents a significant interest for quantum information science.

Recently, various approaches have been proposed for classical and quantum computation with use of microcavity polaritons, see e.g. [5]9. It has been proposed in [5] and then demonstrated in [6] that the classical information can be carried by lower branch (LP) polaritons propagating in microcavity based optical integrated circuits. In quantum domain, an approach for generation of branch-entangled pairs of polaritons in microcavities with use of the spontaneous interbranch parametric scattering has been formulated [7] opening up a possibility to use this process for quantum information processing 8 .

The main advantage of using exciton-polaritons for quantum information processing purposes comes from their fast switching properties (the typical switching time of a few picoseconds), relatively strong nonlinear response, low power to perform logical operations [6] and features of superfluid propagation, which are essential for realization of many algorithms in quantum information science, see e.g. [10, 11]. Current polaritonic devices could be designed by using very well developed semicon- ductor micro- and nanotechnologies and enable to operate at high temperatures, up to the room temperature 12. However, due to open and non-equilibrium nature of the polariton condensates, they cannot serve directly as qubits, since it is not possible so far to generate a polariton state with a fixed well-defined number of particles.

In practice, macroscopic polaritonic system could be used for continuous variable quantum computation, or for quantum comutation with macroscopic polariton states, cf. 13, 14. Light-matter interaction in microcavities gives rise to the natural two-level system, a Rabi doublet, or a doublet of lower, $|L P\rangle$, and upper, $|U P\rangle$, macroscopically occupied orthogonal polariton states

$$
|L P\rangle=C_{x}|X\rangle-C_{p}|P\rangle, \quad|U P\rangle=C_{p}|X\rangle+C_{x}|P\rangle,
$$

being hybridized states of the quantum well exciton, $|X\rangle$, and cavity photon, $|P\rangle$. Hopfield coefficients $C_{x, p}=$ $2^{-1 / 2}\left(1 \pm \Delta / \sqrt{4 g^{2}+\Delta^{2}}\right)^{1 / 2}$ are determined by the system parameters with $g$ being the exciton-photon coupling parameter and $\Delta$ being the detuning between the bare photon and exciton mode and can be controlled in the state-of-the-art structures with needed accuracy. The quantum state of a qubit $|\Psi\rangle$ can be presented as a linear combination of $|L P\rangle$ and $|U P\rangle$ states,

$$
|\Psi\rangle=\beta_{1}|U P\rangle+\beta_{2}|L P\rangle,
$$

with two complex coefficients $\beta_{1,2}$ satisfying the normalization condition $\left|\beta_{1}\right|^{2}+\left|\beta_{2}\right|^{2}=1$. Hence, the quantum state of a qubit is determined by the occupations of the upper and lower polariton states given by $\left|\beta_{1,2}\right|^{2}$, respectively, as well as by their relative phase. In the case of free evolution of the system the coefficients in Eq. (1) read: $\beta_{1,2}=e^{-i \Omega_{1,2} t} / \sqrt{2}$, where $\Omega_{1,2}$ are the eigenfrequencies of UP and LP states, respectively. The beats between LP and UP states are known as Rabi oscillations.

Current progress in the microcavity growth technology makes it possible to produce structures where such Rabioscillator based qubits can be coupled to each other thus 


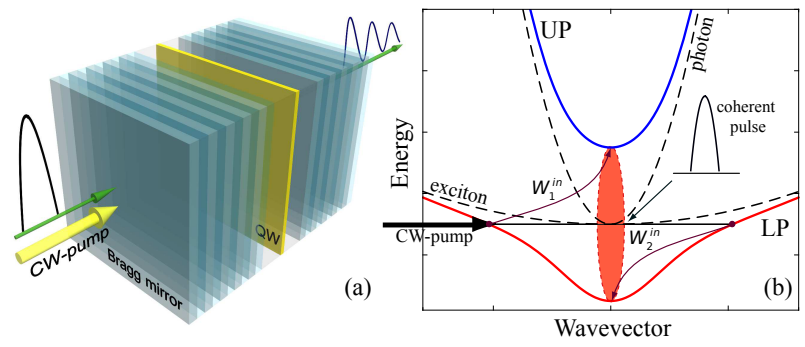

FIG. 1: (Color online) (a) Scheme of polariton qubit excitation in semiconductor microcavity with embedded quantum well (QW) sample. (b) Polariton dispersion and schematic polariton scattering processes supported by incoherent reservoir pumping (shadow area) for $\Delta=0$.

paving way to polariton-based quantum computation devices. However, the main problem here is to preserve a coherence between $|U P\rangle$ and $|L P\rangle$ states within the time of quantum logic operations, or, most generally, during the time of computation [10. Actually, decoherence occurring due to the interaction of the qubit system with its environment prevents application of quantum algorithms [15]. As a result, in the presence of decoherence a polariton qubit state $(2)$ decays as $|\Psi\rangle \propto e^{-t / \tau_{R}}$, where $\tau_{R}$ is characteristic decay time governed by the scattering of polaritons to the reservoir and the photon leakage through the mirrors. The decoherence time may be quite short in realistic systems, typically comparable or even shorter than the lifetime of exciton-polariton, $\tau_{0}$, that is on the picosecond timescale [16. Thus, only a few periods of polariton Rabi-oscillations could have been observed experimentally [18, 19].

The aim of this Letter is to indicate the way of creating of a stable polariton qubit in a resonantly $c w$ pumped system of exciton-polaritons, where Rabi oscillations are induced by a short pulse of light. We demonstrate that in the presence of an incoherent reservoir of polaritons the coherence time of Rabi oscillations may be dramatically increased. This is because of the stimulated scattering of polaritons towards the qubit state $|\Psi\rangle$, which supports the given superposition of $|L P\rangle$ and $|U P\rangle$ states. In realistic microcavity systems the enhancement of the coherence time up to nanoseconds can be achievable.

Model. - We consider exciton-polaritons in a planar or pillar microcavity under incoherent nonresonant $c w$ pumping, see Fig. 1. The pumping helps forming a reservoir of long-living exciton-polaritons with large in-plane wavevectors. We assume that the pumping is strong enough so that only two polariton states $|L P\rangle$ and $|U P\rangle$ can be macroscopically occupied. The dynamics of the Rabi doublet can be most conveniently described by the density matrix approach. The diagonal elements of a $2 \times 2$ density matrix $\varrho$ determine the mean occupations $\varrho_{11}=N_{1}$ of the $|U P\rangle$ and $\varrho_{22}=N_{2}$ of the $|L P\rangle$ states, while off-diagonal elements $\varrho_{12}=\varrho_{21}^{*}$ determine the co- herence between those states. For a pure quantum state in a form of Eq. (2) $\varrho_{11}=\left|\beta_{1}\right|^{2}, \varrho_{22}=\left|\beta_{2}\right|^{2}, \varrho_{12}=\beta_{1} \beta_{2}^{*}$ and $\varrho_{21}=\beta_{1}^{*} \beta_{2}$. In this Letter, we refrain from the analysis of the full statistics of the polariton Rabi doublet, which can be treated by other methods [17, 20, and follow the pseudospin approach like in Ref. [21. For simplicity we neglect polariton-polariton interactions for the macroscopically occupied states. The dynamics of the density matrix is given by $\frac{d \varrho}{d t}=-\frac{i}{\hbar}[\mathcal{H}, \varrho]+\mathcal{L}\{\varrho\}$, where $\mathcal{H}$ is the Hamiltonian of the system whose only nonzero elements are the diagonal ones, $\mathcal{H}_{11}=E_{1}, \mathcal{H}_{22}=E_{2}$, being energies of $|U P\rangle$ and $|L P\rangle$ states, and $\mathcal{L}\{\varrho\}$ stands for the Lindblad superoperator describing dissipation in the system. Equations describing occupation dynamics have a standard form:

$$
\dot{\varrho}_{i i}=\dot{N}_{i}=-\frac{N_{i}}{\tau_{i}}+\left(1+N_{i}\right) W_{i}^{\text {in }}-N_{i} W_{i}^{\text {out }}, \quad i=1,2,
$$

where dots denote time derivatives and $\tau_{i}$ is the lifetime of the $i$ th state. In particular, $\tau_{1,2}$ are expressed through exciton $\left(\tau_{\text {exc }}\right)$ and photon $\left(\tau_{p h}\right)$ lifetimes as $\tau_{1,2}^{-1}=\left|C_{p, x}\right|^{2} \tau_{e x c}^{-1}+\left|C_{x, p}\right|^{2} \tau_{p h}^{-1}$. For the state-of-the-art semiconductor microcavities where $\tau_{\text {exc }} \sim 100 \mathrm{ps}$ and $\tau_{p h} \sim 10$ ps the inequality $\tau_{p h} \ll \tau_{\text {exc }}$ typically holds, cf. [16. In this case one can set $\tau_{1,2} \simeq \tau_{p h} /\left|C_{x, p}\right|^{2}$ for LP and UP branch lifetimes, respectively. In Eqs. (3), $W_{i}^{\text {in }}\left(W_{i}^{\text {out }}\right)$ is the in-scattering rate to (out-scattering rate from) the state $i$ from (to) the reservoir, Fig. 1 $\mathrm{b}$. In general, $W_{1}^{\text {in/out }} \neq W_{2}^{\text {in/out }}$ owing to the significant Rabi splitting $\hbar \Omega_{R}=\left|E_{1}-E_{2}\right|=\hbar \sqrt{\Delta^{2}+4 g^{2}}$, which can be comparable with the energy of exciton-polaritons in the reservoir. As follows from Eqs. (3) any fluctuation $\delta N_{i}=N_{i}-\bar{N}_{i}$, where $\bar{N}_{i}$ are the steady occupations of polariton states, decays according to $\delta N_{i}=-\delta N_{i} / \tau_{c, i}$, where $\tau_{c, i}=\left(\bar{N}_{i}+1\right)\left(1 / \tau_{i}+W_{i}^{\text {out }}\right)^{-1}$, which is the longer, the larger is the occupation of the state [21].

To address the dynamics of Rabi oscillator it is convenient to parametrize the density matrix using the pseudospin formalism: $\varrho_{11}=N+P_{z}, \varrho_{22}=N-P_{z}, \varrho_{12}=$ $P_{x}-\mathrm{i} P_{y}$. Here $N=\left(N_{1}+N_{2}\right) / 2$ and $\boldsymbol{P}=\left(P_{x}, P_{y}, P_{z}\right)$ is the pseudospin. Following [21, 23] we obtain

$$
\begin{gathered}
\dot{N}=-\left[\tau_{+}^{-1}-\delta W_{+}\right] N-\left[\tau_{-}^{-1}-\delta W_{-}\right] P_{z}+W_{+} \\
\dot{P}_{z}=-\left[\tau_{+}^{-1}-\delta W_{+}\right] P_{z}-\left[\tau_{-}^{-1}-\delta W_{-}\right] N+W_{-} \\
\dot{P}_{\perp}=-\left[\tau_{+}^{-1}+\tau_{\perp}^{-1}-\delta W_{+}\right] \boldsymbol{P}_{\perp}-\left[\boldsymbol{\Omega}_{R} \times \boldsymbol{P}_{\perp}\right]
\end{gathered}
$$

Here $\boldsymbol{P}_{\perp}=\left(P_{x}, P_{y}\right), \boldsymbol{\Omega}_{R}=\Omega_{R} \boldsymbol{e}_{z}$, where $\boldsymbol{e}_{z}$ is a unit vector along $z$ axis, $W_{ \pm}=\left(W_{1}^{\text {in }} \pm W_{2}^{\text {in }}\right) / 2, \delta W_{ \pm}=$ $\left[\left(W_{1}^{\text {in }}-W_{1}^{\text {out }}\right) \pm\left(W_{2}^{\text {in }}-W_{2}^{\text {out }}\right)\right] / 2$, and $1 / \tau_{\perp}$ is the additional damping rate for the off-diagonal density matrix components. We stress that the pseudospin $\boldsymbol{P}$ is equivalent to the Bloch vector used to describe the state of any two-level system. In Eqs. (4) we have introduced the characteristic decay rates $\tau_{ \pm}^{-1}=\left(\tau_{1}^{-1} \pm \tau_{2}^{-1}\right) / 2$.

Results and discussion. Figure 2 shows the temporal dynamics of the normalized pseudospin Bloch vector 


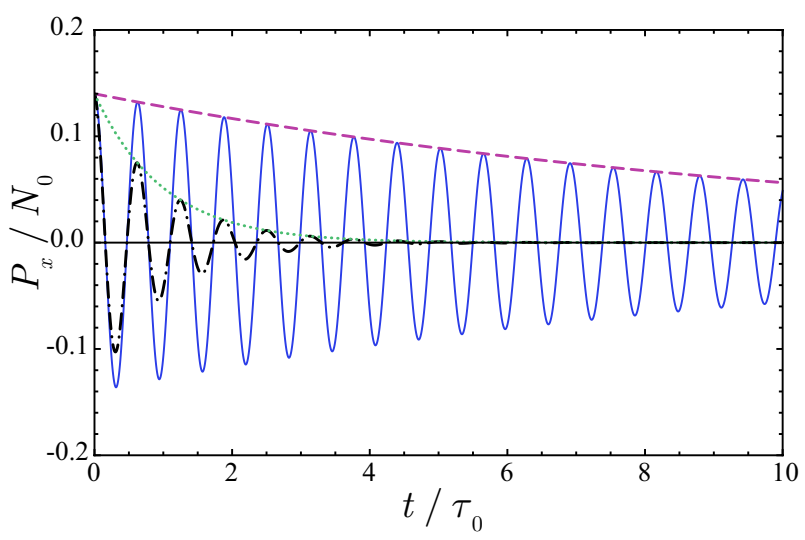

FIG. 2: (Color online) Temporal dynamics of normalized Bloch vector component $P_{x} / N_{0}\left(N_{0} \equiv N(t=0)\right)$ in the presence (solid/blue curve) and in the absence (dash-dotted/black curve) of pumping. Dashed/purple and dotted/green curves show the envelope of Rabi oscillations in the presence and absence of pumping, respectively. The parameters are: $P \tau_{0}=$ $20, W_{1,2}^{\text {out }}=0, \Omega_{R} \tau_{0}=10$. Initial conditions at $t=0$ are: $P_{x} / N_{0}=0.14, P_{y} / N_{0}=0$ and $P_{z} / N_{0}=0.48$.

component $P_{x}$ calculated numerically from Eqs. (4) assuming that $\tau_{\perp} \gg \tau_{0}$ and $C_{x, p}=1 / \sqrt{2}, W_{1}^{\text {in }}=W_{2}^{\text {in }}$. Other parameters of the calculation are presented in the caption to Fig. 2. We assumed that at $t=0$ the coherence between the upper and lower polariton branches is established by a short and weak laser pulse (see Fig. 1), that sets the initial conditions in Fig. 2. As it is clearly seen from Fig. 2, the lifetime of Rabi oscillations $\tau_{R}$ increases with the $c w$ pumping intensity. In such a case, the Rabi oscillations are sustained by the reservoir.

To evaluate this effect analytically, we note that in accordance with Eqs. (4) $\boldsymbol{P}_{x}+i \boldsymbol{P}_{y} \propto e^{-i \Omega_{R} t-t / \tau_{R}}$ where the decay rate of Rabi oscillations $\tau_{R}^{-1}$ is given by

$$
\tau_{R}^{-1}=\tau_{0}^{-1}-\delta W_{+}
$$

Here $\tau_{0}=\tau_{+} \tau_{\perp} /\left(\tau_{+}+\tau_{\perp}\right)$ is the effective lifetime of the polaritonic system without reservoir. It is noteworthy that $\delta W_{+}>0$ means that the incoming scattering rate from the reservoir to the Rabi qubit exceeds the outgoing rate from the qubit to the reservoir, $\tau_{R}>\tau_{0}$. Hence, the Rabi oscillations, which in the pseudospin language are described as a precession of a $\boldsymbol{P}_{\perp}$ around $z$ axis, decay the slower, the higher incoming scattering rate and, hence, the occupation of the ground state are. The population imbalance $P_{z}$ decays with a different time constant $\tau_{r e l}=$ $\tau_{+} /\left(1-\delta W_{+} \tau_{+}\right)$.

The decay rate of Rabi oscillations can be recast in a different form in order to demonstrate that $\tau_{R}>0$ despite of the negative sign in Eq. (5). We introduce the incoming and outcoming scattering rates as: $W_{1,2}^{\text {in }}=$ $\mathcal{W}_{1,2}^{\text {in }} N_{R}, W_{1,2}^{\text {out }}=\mathcal{W}_{1,2}^{\text {out }}\left(1+N_{R}\right)$, where $\mathcal{W}_{i}^{\text {in } / \text { out }}(i=1,2)$ are some constants and the occupation of the reservoir $N_{R}$ must satisfy the following equation:

$$
\dot{N}_{R}=P-\sum_{i=1,2}\left[\mathcal{W}_{i}^{\text {in }}\left(1+N_{i}\right) N_{R}-\mathcal{W}_{i}^{\text {out }} N_{i}\left(1+N_{R}\right)\right]
$$

Here $P$ is the particle generation rate in the reservoir. In what follows we assume that the outscattering processes to the reservoir can be neglected $\left(\mathcal{W}_{1,2}^{\text {out }}=0\right)$ to simplify the subsequent computations. Thus, in the steady-state,

$$
N_{i} \tau_{i}^{-1}-N_{i} N_{R} \mathcal{W}_{i}^{\text {in }}=N_{R} \mathcal{W}_{i}^{\text {in }} \quad i=1,2,
$$

and making use of Eqs. (5), (4a), (6) and the definition of $\delta W_{+}$we obtain

$$
\tau_{R}^{-1}=\tau_{\perp}^{-1}+N_{R}\left(\mathcal{W}_{1}^{\text {in }} N_{1}^{-1}+\mathcal{W}_{2}^{\text {in }} N_{2}^{-1}\right) / 2>0 .
$$

It is clearly seen that an increase of the occupation of the doublet lease to the decrease of $1 / \tau_{R}$ and the increase of the coherence time of Rabi oscillations. The closed form result for the $\tau_{R}$ dependence on the pumping rate can be obtained taking into account that the steady-state occupancy of the reservoir can be found from the following equation

$$
P=\frac{\mathcal{W}_{1}^{\text {in }} N_{R}}{1-\tau_{1} \mathcal{W}_{1}^{\text {in }} N_{R}}+\frac{\mathcal{W}_{2}^{\text {in }} N_{R}}{1-\tau_{2} \mathcal{W}_{2}^{\text {in }} N_{R}} .
$$

Finally, we obtain

$$
\begin{gathered}
\tau_{R}{ }^{-1}=\tau_{0}{ }^{-1}-\frac{1+\alpha}{4 \alpha} \frac{P\left(\alpha \tau_{1}+\tau_{2}\right)+\alpha+1-}{P \tau_{1} \tau_{2}+\tau_{2}+\tau_{1}} \\
\frac{-\sqrt{P^{2}\left(\alpha \tau_{1}-\tau_{2}\right)^{2}+2 P\left(\alpha \tau_{1}-\tau_{2}\right)(\alpha-1)+(\alpha+1)^{2}}}{P \tau_{1} \tau_{2}+\tau_{2}+\tau_{1}}
\end{gathered}
$$

where $\alpha=\mathcal{W}_{1}^{\text {in }} / \mathcal{W}_{2}^{\text {in }}$ and it is assumed that $0 \leq \alpha \leq 1$. For $\alpha>1$ in Eq. 10 one has to replace $\alpha \rightarrow 1 / \alpha$ and exchange $\tau_{1}$ and $\tau_{2}$.

Figure 3 shows the dependence of $\tau_{0} / \tau_{R}$ on $P \tau_{0}$ calculated for various values of $\alpha$. Here, similarly to Fig. 2 we consider $\left|C_{x, p}\right|^{2}=1 / 2$ that implies equal upper and lower branch polariton lifetimes, i.e. $\tau_{p o l} \equiv \tau_{1}=\tau_{2}$. Experimentally, this condition can be verified in specially designed pillar microcavities 22], while in planar cavities the lifetime of a $|U P\rangle$ state is usually much shorter than the lifetime of an $|L P\rangle$ state. The imbalance of lifetimes in this case can be compensated by the imbalance of pumping, which may be achieved in the case of a quasiresonant pumping of the $|U P\rangle$ state and can be accounted for in our model by a proper choice of the parameter $\alpha$. We shall also assume $\tau_{\perp} \gg \tau_{0}$. In this limit, the effective lifetime of the polaritonic system $\tau_{0}$ approaches to $\tau_{0} \simeq \tau_{p o l}$. If, by contrast, $\tau_{\perp}$ is comparable with $\tau_{p o l}$ than at $P \tau_{\text {pol }} \rightarrow \infty$

$$
\frac{\tau_{p o l}}{\tau_{R}} \simeq \frac{\tau_{p o l}}{\tau_{\perp}}+\frac{1-\alpha}{2},
$$




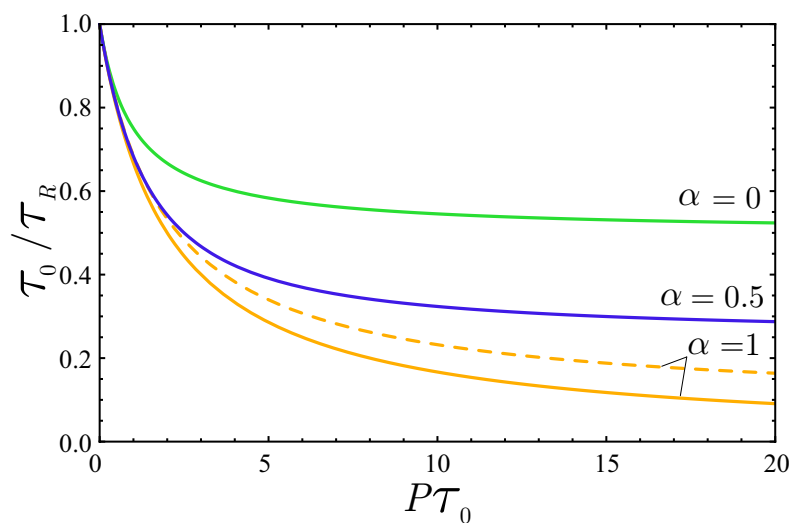

FIG. 3: (Color online) Ratio $\tau_{0} / \tau_{R}$ as a function of the pumping rate $P \tau_{0}$. For solid (dashed) curves $\tau_{1}=\tau_{2}\left(\tau_{2}=10 \tau_{1}\right)$.

meaning that the decay time of Rabi oscillations is limited by $\tau_{\perp}$. In any case, the decay rate of Rabi oscillations decreases with the increase of the pumping rate, but the asymptotic value depends on the imbalance of the scattering rates towards $|L P\rangle$ and $|U P\rangle$ states having its minimum at $\alpha=1$, i.e. $\mathcal{W}_{1}^{\text {in }}=\mathcal{W}_{2}^{\text {in }}$, cf. (11) and Fig. 3 . In the optimum case, which would correspond to a pillar microcavity with equal $|L P\rangle$ and $|U P\rangle$ lifetimes, $\alpha=1$, $\tau_{\perp} \rightarrow \infty$, for $\tau_{p o l}=10 \mathrm{ps}$ and ground state occupation $N=10^{2}$ we have $\tau_{R} \sim 1 \mathrm{~ns}$.

In planar microcavities the lifetimes of $|U P\rangle$ and $|L P\rangle$ states can be strongly different. Importantly, an increase of the coherence time $\tau_{R}$ can be observed in this case as well: see dashed curve in Fig. 3 Particularly, if $\mathcal{W}_{1}^{\text {in }} / \mathcal{W}_{2}^{\text {in }}=\tau_{2} / \tau_{1}$ the populations of upper and lower polariton states become equal and $\tau_{R}=\tau_{0}\left(1+P \tau_{0} / 2\right)$.

Let us study the properties of the polariton qubit described by Eq. (2). Without any loss of generality one can rewrite Eq. (2) as

$$
|\Psi\rangle=e^{-i \omega_{0} t}\left[\cos \left(\frac{\theta}{2}\right)|0\rangle+e^{i \pi / 2} \sin \left(\frac{\theta}{2}\right)|1\rangle\right],
$$

where we introduced the azimuthal angle $\theta=\Omega_{R} t$, states $|0\rangle=\left(e^{-i \varphi}|L P\rangle+|U P\rangle\right) / \sqrt{2}$ and $|1\rangle=$ $\left(e^{-i \varphi}|L P\rangle-|U P\rangle\right) / \sqrt{2}$ represent orthogonal (computational) qubit states with $\varphi$ being an arbitrary phase, $\omega_{0}=\left(\Omega_{p h}+\Omega_{e x c}\right) / 2, \Omega_{p h}$ and $\Omega_{e x c}$ being the bare frequencies of the photon and exciton modes, respectively.

Figure 4 shows the time evolution of the qubit state $|\Psi\rangle$ for various ratios of $\tau_{0} / \tau_{R}$ at the Bloch sphere. Since one of the Euler angles is equal to $\pi / 2$, see Eq. (12), the qubit Bloch vector evolves in a plane. In particular, it is clearly seen that due to the reservoir supported Rabi oscillations the decoherence effects are essentially suppressed for the solid (red) trajectory.

The manipulation of the qubit state Eq. 12 can be tailored through variation of phase for a fixed Rabi frequency $\Omega_{R}$. On the other hand, it is possible to manipulate by $\Omega_{R}$ as well by controlling the exciton-photon

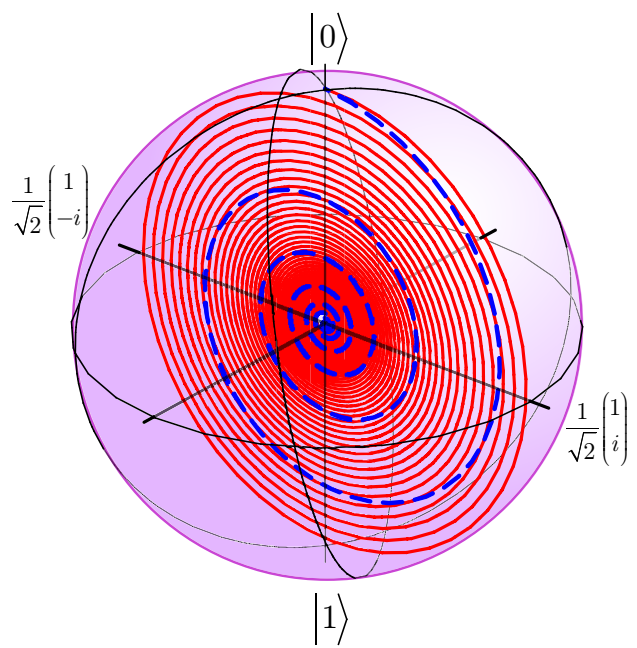

FIG. 4: (Color online) Bloch sphere representation of polariton qubit dynamics without (dashed curve) and with (solid curve) reservoir supported Rabi oscillations. The parameters are $\alpha=1, \varphi=\pi / 2, P \tau_{0}=20$.

coupling parameter $g$ by external electric fields, which affect the exciton oscillator strength, or by manipulating the exciton-photon detuning $\Delta$, cf. 24. In the latter case, however, the Hopfield coefficients $C_{p}$ and $C_{x}$ also vary and the states $|0\rangle$ and $|1\rangle$ are no more basic computational states of the system. Hence, it is instructive rewrite Eq. 12. in the exciton-photon basis in this case. In particular, from Eqs. (1), 12 we express $|\Psi\rangle=e^{-i \omega_{0} t}\left[\beta_{1}|X\rangle+\beta_{2}|P\rangle\right]$, where the coefficients $\beta_{1,2}$ are defined as $\beta_{1,2}=\left(e^{-i \theta / 2} C_{p, x} \pm e^{i \theta / 2-i \varphi} C_{x, p}\right) / \sqrt{2}$, cf. Eq. (2). In this form the qubit state $|\Psi\rangle$ represents a linear superposition of matter (excitonic) and photonic qubit state. The phase $\varphi$ determines the initial state of the qubit. The short pumping pulse sets the initial condition of $\beta_{2}=1$ that corresponds to the purely photonic state $|\Psi(t=0)\rangle=|P\rangle$.

Next, for quantum information applications, it is important to demonstrate the entanglement between different qubit states. Such an entanglement could be achieved with use of the coupled cavity architecture, cf. [25, 26. In this case, the entanglement can be achieved due to the photonic tunneling between neighboring cavities.

Polarton qubits can be used for quantum cloning and quantum memory applications [27]. In particular, the quantum cloning procedure of photonic state onto UP and LP states can be realized by using an algorithm proposed by some of the present authors in [9] which implies the coherent manipulation of Hopfield coefficients.

Furthermore, the dynamical memory algorithm (see e.g. 28]) can be realized using the semiconductor microcavity structures described above. This algorithm imposes mapping of the quantum information contained in the initially prepared photonic state $|\Psi(t=0)\rangle=|P\rangle$ onto the excitonic qubit state $|X\rangle$. Writing, reading and 
storage stages in this case can be achieved by a time control of the exciton dipole matrix element [parameter $g \equiv g(t)]$ and/or by manipulation of the exciton-photon detuning $\Delta \equiv \Delta(t)$ adiabatically, cf. 24]. We expect that the proposed mechanism of enhancement of Rabi oscillations would allow realization of high temperature quantum memories with a lifetime of the order of hundred of picoseconds.

Acknowledgments. The financial support from the Russian Ministry of Education and Science (Contract No. 11.G34.31.0067 with SPbSU and leading scientist A. V. Kavokin), RFBR and EU projects POLAPHEN, SPANGL4Q and LIMACONA is acknowledged. This work is also supported by the Russian Ministry of Education and Science state task 2014/13. A. P. Alodjants acknowledges support from "Dynasty" Foundation.

[1] T. Feurer, N. S. Stoyanov, D. W. Ward, et al., Annu. Rev. Matter. Res. 37, 317 (2007).

[2] M. Fleischhauer, M. D. Lukin, Phys. Rev. A 65, 022314 (2002).

[3] M. Y. Hartmann, F. G. S. L. Brandao, M. B. Plenio, Nat. Phys. 2, 849 (2006); ibid A. D. Greentree, C. Tahan, Y. H. Cole, L. Hollenberg, 856.

[4] I. Yu. Chestnov, A. P. Alodjants, S. M. Arakelian, et al., Phys. Rev. A 85, 053648 (2012); ibid E. S. Sedov, A. P. Alodjants, S. M. Arakelian, et al., 84, 013813 (2011).

[5] C. Leyder, T. C. H. Liew, A. V. Kavokin, et al., Phys. Rev. Lett. 99, 196402 (2007), ibid T. C. H. Liew, A. V. Kavokin, and I. A. Shelykh, Phys. Rev. Lett. 101, 016402 (2008).

[6] D. Ballarini, M. De Giorgi, E. Cancellieri, et al., Nature Comms 4, 1778 (2013); M. De Giorgi, D. Ballarini, E. Cancellieri et al., Phys. Rev. Lett. 109, 266407 (2012).

[7] C. Ciuti, Phys. Rev. B 69, 245304 (2004); see also N. A. Gippius, S. G. Tikhodeev, V. D. Kulakovskii, D. N. Krizhanovskii and A. I. Tartakovskii, EPL 67, 997 (2004).

[8] D. Pagel and H. Fehske, J. Sperling and W. Vogel, Phys. Rev. A 86, 052313 (2012)

[9] A. P. Alodjants, S. M. Arakelian, S. N. Bagayev, I. A. Chekhonin, V. S. Egorov, J. of Rus. Laser Res. 27, 482 (2006).
[10] M. A. Nielsen and Isaac L. Chuang, Quantum Computation and Quantum Information (Cambridge University Press, 2010).

[11] T. D. Ladd, F. Jelezko, R. Laflamme, Y. Nakamura, C. Monroe, J. L. O'Brien, Nature 464, 45 (2010).

[12] S. Christopoulos, G. Baldassarri Höger von Högersthal, A. J. D. Grundy, P. G. Lagoudakis, A. V. Kavokin, J. J. Baumberg, G. Christmann, R. Butté, E. Feltin, J.-F. Carlin, and N. Grandjean, Phys. Rev. Lett. 98, 126405 (2007).

[13] M. C. de Oliveira and W. J. Munro, Phys. Rev. A 61, 042309 (2000); ibid B. C. Sanders, 45, 6811 (1992).

[14] I-H. Chen, Y. Y. Lin, Y.-C. Lai, E. S. Sedov et al., Phys. Rev. A 86, 023829 (2012).

[15] G. M. Palma, K.-A. Suominen and A. K. Ekert, Proc. R. Soc. Lond. A 452, 567 (1996).

[16] V. Timofeev, D. Sanvitto (Eds.), Exciton Polaritons in Microcavities (Springer, Berlin, 2012).

[17] F. P. Laussy Quantum Dynamics of Polariton Condensates in Exciton Polaritons in Microcavities ed. by V. Timofeev, D. Sanvitto (Springer, 2012).

[18] J.D. Berger, O. Lyngnes, H.M. Gibbs, G. Khitrova, T.R. Nelson, E.K. Lindmark, A.V. Kavokin, M.A. Kaliteevski, and V.V. Zapasskii, Phys. Rev. B 54, 1975 (1996).

[19] A. Brunetti, M. Vladimirova, D. Scalbert, M. Nawrocki, A. V. Kavokin, I. A. Shelykh, and J. Bloch, Phys. Rev. B 74, 241101 (2006).

[20] D. Read, T. C. H. Liew, Y. G. Rubo, and A. V. Kavokin, Phys. Rev. B 80, 195309 (2009).

[21] M. M. Glazov, M. A. Semina, E. Y. Sherman, and A. V. Kavokin, Phys. Rev. B 88, 041309 (2013).

[22] M. Maragkou, A. J. D. Grundy, E. Wertz, et al., Phys. Rev. B 81, 081307R (2010)

[23] K. V. Kavokin, I. A. Shelykh, A. V. Kavokin, G. Malpuech, and P. Bigenwald, Phys. Rev. Lett. 92, 017401 (2004).

[24] E. S. Sedov, A. P. Alodjants, S. M. Arakelian, et al., arXiv:1312.2536 A. P. Alodjants, S. M. Arakelian, and A. Yu. Leksin, Laser Physics 17, 1432 (2007).

[25] C.-H. Su, A. D. Greentree, W. J. Munro, K. Nemoto, L. C. L. Hollenberg, Phys. Rev. A 78, 062336 (2008).

[26] G. Panzarini, L. C. Andreani, A. Armitage, et al., Phys. Rev. B 59, 5082 (1999).

[27] C. Simon, M. Afzelius, J. Appel, et al., Eur. Phys. J. D 58, 1 (2010);

[28] K. Heshami, A. Green, Y. Han, et al., Phys. Rev. A 86, 013813 (2012); ibid Q. Y. He, M. D. Reid, E. Giacobino, et al., 79, 022310 (2009). 